\title{
The Benefit of Benralizumab Monoclonal Antibody Treatment for Severe Eosinophilic Asthma in a Case Series (Pulmonology Clinic Târgu Mureș, Romania)
}

Nimród László*1, Hédy Katalin Sárközy², Cristina Alexandra Man*1, Edith Simona lanoși², Botond Mátyás², Bianca Emilia Ciurba1, Corina Mărginean¹, Gabriela Jimborean²

${ }^{1}$ Clinic of Pneumology, Emergency County Clinical Hospital, Târgu Mureș, Romania

2 "George Emil Palade" University of Medicine, Pharmacy, Science and Technology, Târgu Mureș, Romania

*These two authors have equal contribution to the article

\section{CORRESPONDENCE}

\section{Hédy Katalin Sárközy}

Str. Gheorghe Marinescu nr. 5

540098 Târgu Mures, Romania

Tel: +40 265230001

E-mail: baloghedikatalin@yahoo.com

\section{ARTICLE HISTORY}

Received: September 4, 2021

Accepted: September 11, 202

Nimród László • Str. Gheorghe Marinescu nr. 5 540098 Târgu Mureș, Romania. Tel: +40 730563 544, E-mail: laszlonimrod@yahoo.com

Cristina Alexandra Man • Str. Gheorghe Marinescu nr. 5, 540098 Târgu Mureș, Romania. Tel: +40 265230 001,E-mail:man_cristina_alexandra@yahoo.com

Bianca Emilia Ciurba • Str. Gheorghe Marinescu nr 5, 540098 Târgu Mureș, Romania. Tel: +40 748465 469, E-mail: biancaciurba@outlook.com

Edith Simona lanoși • Str. Gheorghe Marinescu nr. 5, 540098 Târgu Mures, Romania. Tel: +40 265230 001, E-mail: ianosi_edith70@yahoo.com

Botond Mátyás - Str. Gheorghe Marinescu nr. 38, 540036 Târgu Mures, Romania. Tel: +40 265215 551, E-mail: mukus44@gmail.com

Corina Mărginean • Str. Gheorghe Marinescu nr. 5 540098 Târgu Mures, Romania. Tel: +40 265230 001, E-mail: marginean_corina@yahoo.com

Gabriela Jimborean • Str. Gheorghe Marinescu nr. 5, 540098 Târgu Mureș, Romania. Tel: +40 265230 001, E-mail: gabriela jimborean275@gmail.com

\begin{abstract}
Background: Monoclonal antibody therapy is currently an additional treatment option to reduce exacerbations and improve symptom control in patients with severe eosinophilic asthma (SEA) that is uncontrolled despite treatment with high-dose inhaled corticosteroids and longacting beta- 2 agonists. Benralizumab, a monoclonal antibody that binds to the interleukin- 5 receptor (IL-5), significantly reduces symptoms and annual exacerbations, as well as the use of systemic corticosteroids in patients with SEA. However, few studies are available on the effectiveness of this biological treatment in real life. The aim of this case series was to evaluate the efficacy of benralizumab by analyzing changes in clinical parameters and blood eosinophils in patients with SEA. Methods: We analyzed four patients with SEA who started treatment with benralizumab. The history of symptoms and exacerbations, eosinophil counts, data regarding the oral corticosteroid dose, need for rescue treatment, spirometry measurements and asthma control questionnaires (ACT) regarding the level of asthma control were recorded. A positive response to treatment was defined by a significant reduction in eosinophil counts, increased ACT scores, and lower rates of exacerbations. Results and conclusions: Benralizumab monoclonal antibody was effective in all four patients. This was shown by a reduction in exacerbation rates, symptom severity, and lower dose of oral corticosteroids and rescue medication. This novel treatment was well tolerated by the analyzed patients, thus indicating that benralizumab is an attractive choice for patients due to eosinophilic count reduction as well as the less frequent dosing schedule. However, further studies are required, on larger populations.
\end{abstract}

Keywords: monoclonal antibody therapy, severe eosinophilic asthma, clinical parameters, eosinophils 


\section{INTRODUCTION}

Severe eosinophilic asthma (SEA) is defined by remaining uncontrolled despite maximum optimal treatment (with good adherence and inhalation technique but also with the control of contributing factors) in advanced stages, or worsening symptoms despite treatment with inhaled corticosteroids. ${ }^{1}$ About $3-10 \%$ of people with asthma have SEA. ${ }^{2}$

SEA carries a great burden on affected patients, with a significant decrease in the quality of life due to frequent exacerbations, disability, and increased mortality rates. In addition, SEA is also a social and economic problem for patients and their families due to increased healthcare costs related to treatment, frequent emergency presentations, imaging and laboratory testing, but also indirectly due to temporary or permanent loss of working ability. ${ }^{3}$

Monoclonal antibodies, such as benralizumab, are among the newest therapeutic options for severe asthma. This medication is added to general measures (education, preventive measures for exacerbations) and to the daily maintenance therapy. Monoclonal antibodies have longterm beneficial effects including increased symptoms control, reduced use of systemic corticosteroids and rescue medication, and decreased number and severity of exacerbations. Benralizumab has shown therapeutic benefits in patients over 12 years of age and in adults with SEA that is not adequately controlled by inhaled high-dose corticosteroids in combination with long-acting beta-agonists. ${ }^{4}$

Benralizumab binds to the alpha subunit of the IL-5 receptor (from eosinophils or basophils involved in asthmatic inflammatory response), thus leading to cell apoptosis and cell death by attracting natural killer T cells. This action significantly reduces the asthma inflammatory response mediated by eosinophils. Benralizumab has effects on symptom control, reduced exacerbations, improved lung function and quality of life in patients with SEA.., 6

Benralizumab is administered by subcutaneous injection (30 mg) every 4 weeks (first 3 doses) and then 1 dose every 8 weeks. Studies have shown that one intravenous dose and several subcutaneous ones reduce the number of eosinophils in the bronchial mucosa/submucosa, but also in the bone marrow and peripheral blood. ${ }^{6}$

The depletive effect of blood eosinophils is established within the first 24 hours after the first dose, and the effect is maintained throughout the treatment period. The depletion of blood eosinophils is accompanied by a reduction in eosinophilic granular proteins - eosinophil-derived neurotoxin (EDN) and eosinophilic cationic protein (ECP) but also by a reduction in the number of blood basophils. ${ }^{7}$
Eligibility criteria for benralizumab include patients with SEA (correctly treated in steps $4-5$ of the GINA classification), with multiple exacerbations in the last year and elevated peripheral blood eosinophil count $\geq 300$ elements $/ \mu \mathrm{L}$ ). Patients taking oral corticosteroids (OCS) with corticoid-dependent asthma are a well-indicated group given the known risk of long-term side effects of systemic corticosteroids. ${ }^{8}$

The aim of the manuscript was to conduct a case series study to evaluate the effect of benralizumab on patients with SEA, despite optimal medical treatment, by analyzing eosinophil blood count and the patients' quality of life.

\section{MATERIALS AND METHODS}

We prospectively analyzed four SEA patients who were under observation and treatment in the Pulmonology Clinic of Târgu Mureș, Romania, in 2019. The results of the present manuscript illustrate the preliminary results of a larger study conducted in 2019. Inclusion criteria were the following: age over 18 years and severe asthma which remains uncontrolled despite maximum optimal treatment, with elevated blood eosinophil count $\geq 300$ elements $/ \mu \mathrm{L}$ and multiple exacerbations in the last year. Exclusion criteria consisted of patients under 18 years, blood eosinophils $<300$ elements/ $\mu \mathrm{L}$, Asthma Control Questionnaire (ACT) score over 21 . The study was conducted in accordance with the principles stipulated in the Declaration of Helsinki. All subjects agreed with processing of their data, and the study procedures were carried out following approval of the institution where the patients were treated.

\section{CASE SERIES}

\section{Case no. 1}

We present the case of a 68 -year-old patient, former smoker (34 packs/year), with a body mass index (BMI) of $26.14 \mathrm{~kg} / \mathrm{m}^{2}$, known with hypertension, previous stroke, chronic asthma, and allergic rhinitis diagnosed 7 years prior to his current presentation. The patient was under treatment with inhaled fluticasone/salmeterol 500/50 $\mu \mathrm{g}$ 2 times/day, salbutamol $100 \mu \mathrm{g}, 2$ inhalations as needed + tiotropium bromide $2,5 \mu \mathrm{g} / \mathrm{dose}, 2$ inhalations/day in the morning, montelukast sodium $10 \mathrm{mg} 1 \mathrm{tb} /$ day, and intranasal beclomethasone twice daily. The patient presented 3 months prior to the current presentation with altered general state, dyspnea during light exertion, productive cough with mucous and muco-purulent sputum, and excessive sweating. The exacerbation was considered mild and was 
treated at home with oral antibiotics and a short course of OCS. The prescribed medication had improved the general condition but there was a persistence of dyspnea and cough. During the next 2 months, the patient's condition changed, the dyspnea had worsened, became persistent at rest and was associated with nocturnal paroxysmal dyspnea, wheezing, low-grade fever and sweating, which led to the patient's admission to the Pulmonology Clinic. Given the frequent exacerbations, incomplete control of asthma under maximized treatment, and the existence of an increased number of blood eosinophils $\left(3.7 \cdot 10^{3}\right.$ elements/ $\mu \mathrm{L}$ ), initiation of benralizumab $30 \mathrm{mg} /$ dose monthly treatment was prescribed, followed by subcutaneous administration every 8 weeks. The baseline ACT was 14 points, which implicitly suggested the lack of control of the disease. At only 2 hours after starting the treatment with benralizumab, the number of eosinophils decreased to $0.5 \cdot 10^{3}$ elements $/ \mu \mathrm{L}$. The patient had received the second and third dose at intervals of 1 month during which the general condition and dyspnea improved significantly, with no more nocturnal awakenings and the number of eosinophils remained low $\left(0.01 \bullet 10^{3}\right.$ elements $\left./ \mu \mathrm{L}\right)$. The ACT questionnaire recorded 25 points after 2 months (3 doses).

\section{Case no. 2}

This is the case of a 67-year-old female patient, non-smoker, without exposure to respiratory toxins, with a 5 year history of persistent asthma, associated with ischemic heart disease with right bundle branch block and hypertension under treatment. The patient was prescribed inhaled budesonide/formoterol 320/9 $\mu \mathrm{g}$ (2 inhalations/ day) and salbutamol $100 \mu \mathrm{g}$ ( 2 inhalations as needed). Due to persistent symptoms (diurnal dyspnea, wheezing, nocturnal dyspnea in seizures) despite compliance with treatment and the correct technique of administration, the patient needed daily rescue medication with overuse of short-acting beta agonists (SABA) due to lack of disease control. The investigation of the contributing factors for the lack of disease control highlighted an allergic rhinitis for which treatment with mometasone furoate $2 \times 2$ nasal inhalations/day was initiated and exposure to air allergens, food allergens, animals, dust, chemicals were eliminated. Eight months prior, the patient presented to the Pulmonology Clinic with an altered general condition, dyspnea during low exertion, nocturnal wheezing, and cough with mucopurulent sputum. Due to the lack of asthma control with standard treatment (daily and nocturnal symptoms), exacerbation and decreased lung function (moderate ob- structive ventilatory dysfunction), an ACT questionnaire of 12 points, an eosinophil count of $6.2 \cdot 10^{3}$ elements/ $\mu \mathrm{L}$, treatment with benralizumab $30 \mathrm{mg} /$ dose was started with subcutaneous administration. Shortly after the first dose, the symptoms improved visibly with the decrease of cough, wheezing, and the need for SABA. The eosinophil count was $0.2 \cdot 10^{3}$ elements $/ \mu \mathrm{L}$ after the first dose. The patient had received 6 doses of benralizumab, and during this period she did not show exacerbations, the nocturnal symptoms disappeared, the diurnal symptoms were minimal without the use rescue medication (less than 2 times/ week), and the ACT score had increased up to 25 points.

\section{Case no. 3}

A 55-year-old hypertensive male patient, non-smoker, with a 3-year-old diagnosis of asthma on high dose fluticasone/salmeterol treatment 500/50 $\mu \mathrm{g} 2$ times/day and SABA $100 \mu \mathrm{g} 2$ inhalations as needed, addressed to the Pneumology Clinic with exacerbated symptoms. The patient had had in fact an increase in the consumption of rescue medication (around 6 times/day) due to his persistent symptoms (dyspnea at low exertion, wheezing, irritating cough). Functional tests showed an average mixed ventilatory dysfunction and an ACT score of 14 points. The patient had a body mass index of $34 \mathrm{~kg} / \mathrm{m}^{2}$. Blood eosinophil count was $0.4 \cdot 10^{3}$ elements $/ \mu \mathrm{L}, 6.5 \%$ of the total white blood cell count. Exacerbation management required short-term OCS, antibiotic therapy, education to optimize inhaled treatment and to eliminate contributing risk factors (antigen eviction, weight loss, proton pump inhibitors for gastro-esophageal reflux). Inhalation technique and treatment compliance were reevaluated and benralizumab was initiated. At 24 hours after the first dose of benralizumab, the symptoms had improved significantly and the patient did not require any use of rescue medication or OCS. The number of eosinophils had decreased to 0.01 • $10^{3}$ elements $/ \mu \mathrm{L}, 2.8 \%$ of all white blood cells.

\section{Case no. 4}

An 82-year-old patient (former 36 pack/year smoker with chronic obstructive pulmonary disease), known for associated persistent asthma for the last 4 years, with frequent infectious exacerbations, was admitted to the Pulmonology Clinic with dyspnea at low exertion, cough with mucopurulent sputum, chest pain, and wheezing. The chest computed tomography revealed advanced pulmonary emphysema but no other associated pathology. The cardiological work-up revealed signs of chronic pulmonary 
heart disease in the early stages, and the respiratory functional tests showed a mixed reversible ventilatory dysfunction. The patient had undergone inhalator treatment with formoterol/budesonide $9 / 320 \mu \mathrm{g}$ ( 2 inhalations/day), tiotropium bromide $2.5 \mu \mathrm{g} /$ day and salbutamol $100 \mu \mathrm{g}$ (2 inhalations as needed). The ACT questionnaire noted the existence of poor asthma control (12 points), the patient had a body mass index of $27.43 \mathrm{~kg} / \mathrm{m}^{2}$, and blood eosinophilia was increased $\left(3.2 \cdot 10^{3}\right.$ elements $\left./ \mu \mathrm{L}\right)$. Along with the exacerbation treatment (antibiotics, oxygen, mucolytics), the initiation of biological treatment with benralizumab $30 \mathrm{mg}$ subcutaneously was prescribed. Within 2 hours after the first dose, the number of eosinophils had decreased to $0.19 \cdot 10^{3}$ elements $/ \mu \mathrm{L}$ and after 24 hours, the eosinophil count had decreased to $0.09 \bullet 10^{3}$ elements / $\mu \mathrm{L}$. The clinical condition had significantly improved and the use of OCS was avoided. The ACT questionnaire at 2 months had improved significantly in parallel with consistent control of symptoms (decreased persistent dyspnea, nocturnal awakenings, increased daily physical activity, and the disappearance of nocturnal awakenings).

Table 1 illustrates the ACT questionnaire results of all 4 patients included in the present case series before the initiation of benralizumab, as well as after one month and 2 months, respectively. There was a clear increase in the ACT score in all patients.

\section{DISCUSSIONS}

The significant improvement in asthma control in the presented cases showed, on the one hand, the effectiveness, and on the other, the safety of benralizumab therapy after the acute phase of SEA exacerbation, thus indicating an effective and rapid response in symptom improvement and inflammation reduction. Blood eosinophil count had decreased significantly after the first dose (in 2 cases even after 2 hours) and symptoms had improved significantly, with reduced cough, sputum production, and dyspnea.

TABLE 1. The evolution of the ACT questionnaire values in the studied patients before and one to two months after benralizumab initiation

\begin{tabular}{lccc}
\hline Case & $\begin{array}{c}\text { ACT score before } \\
\text { treatment }\end{array}$ & $\begin{array}{c}\text { ACT score after } \\
\text { 1 month of } \\
\text { treatment }\end{array}$ & $\begin{array}{c}\text { ACT score after } \\
\text { 2 months of } \\
\text { treatment }\end{array}$ \\
\hline 1 & 12 & 23 & 25 \\
2 & 13 & 24 & 25 \\
3 & 14 & 24 & 25 \\
4 & 13 & 19 & 22 \\
\hline
\end{tabular}

It is known that the inflammatory response in asthma is associated with increased eosinophils in the bronchial mucosa and blood, which increase additionally during exacerbations, thus leading to poor control of asthma. ${ }^{9}$ In patients with SEA, there is an intense activation of T2-mediated inflammation, leading to an overexpression of IL-5 receptors which decreases the sensitivity of eosinophils to corticosteroids. ${ }^{10}$

The proapoptotic effect of corticosteroids on inflammatory cells decreases greatly if there is an antiapoptotic action caused by elevated levels of IL-5. ${ }^{11}$ Thus, IL-5 is of crucial importance for the development and worsening of eosinophilic asthma associated with type 2 inflammatory immune response. ${ }^{12,13}$

The action of monoclonal antibodies, such as benralizumab, includes the blockade of IL-5 receptors in eosinophils and basophils, and the modulating effect of antibodydependent cell-mediated cytotoxicity. This causes the total depletion of eosinophils in both blood and tissues. Benralizumab also decreases the concentrations of cytokines released by eosinophils (EDN and ECP). ${ }^{14}$

Numerous studies have shown that benralizumab decreases both current asthma symptoms and exacerbations in the first 30 days of treatment. The improvement occurs rapidly through IL-5 binding and decreased asthma-related inflammation. $4,5,8,15$

In the present proof of concept study, we used the Asthma Control Test (ACT) Questionnaire for the subjective assessment of asthma, before and after treatment with benralizumab. The analyzed criteria included the symptom levels within the last month, the frequency of rescue medication use, the effect of asthma on daily activities, and an overall assessment of the degree of asthma control. ${ }^{16}$ All patients had poor control (score below 15) of asthma initially, despite maximized medication which included corticosteroids and long-acting beta-agonists. After initiating treatment (even after 1 dose), according to the ACT questionnaire all patients showed a significant improvement in symptoms and indirectly in the quality of life, with an associated decrease in SABA consumption. Starting from an initial score of 12-13 points, after 2 months, 3 of the patients obtained a score of 25 (maximum number of points) and 1 patient obtained a score of 22, which was explained by the existence of an overlap with smoking and COPD.

This strong impact on asthma control is due to the ability of benralizumab to drastically decrease eosinophils in the peripheral blood and the airways, thus restricting the entry of toxic proteins resulting from degranulation of eosinophils within the bronchial tissue. 


\section{CONCLUSIONS}

The administration of monoclonal antibodies, such as benralizumab, in combination with pre-existing maintenance treatment that includes inhaled corticosteroids and longacting beta-agonists, led to a rapid clinical improvement in all patients. This was shown by a lack of exacerbations, decreased symptoms, and overall improvement in the quality of life. The improvement was correlated with a decrease in the blood eosinophil count. In all patients, the treatment had a very good tolerance, without any side effects. The action of benralizumab was rapid in exacerbated patients, preventing the introduction of systemic corticosteroids. The ACT questionnaire was a useful tool for assessing the level of asthma control and was correlated with the evolution of blood eosinophil count. Benralizumab-type monoclonal antibodies should be integrated into clinical use in patients with incomplete asthma control despite optimal medical treatment. Further studies are required on larger patient sets.

\section{CONFLICT OF INTEREST}

Nothing to declare.

\section{REFERENCES}

1. Kommaraju K, Latifi M. Outpatient management of asthma in adults: A snapshot of the 2020 GINA report. Cleve Clin J Med. 2021;88:377-380.

2. Wang E, Wechsler ME, Tran TN, et al. Characterization of Severe Asthma
Worldwide: Data From the International Severe Asthma Registry. Chest. 2020;157:790-804

3. Burney P, Jarvis D, Perez-Padilla R. The global burden of chronic respiratory disease in adults. Int J Tuberc Lung Dis. 2015;19:10-20.

4. Menzies-Gow A, Corren J, Bel EH, et al. Corticosteroid tapering with benralizumab treatment for eosinophilic asthma: PONENTE Trial. ERJ Open Res. 2019:5:00009-2019.

5. Park HS, Kim MK, Imai N, et al. Asian Benralizumab Study Group. A Phase 2a Study of Benralizumab for Patients with Eosinophilic Asthma in South Korea and Japan. Int Arch Allergy Immunol. 2016;169:135-145.

6. Laviolette M, Gossage DL, Gauvreau G, et al. Effects of benralizumab on airway eosinophils in asthmatic patients with sputum eosinophilia. Allergy Clin Immunol. 2013;132:1086-1096.

7. Bagnasco D, Heffler E, Testino E, Passalacqua G, Canonica GW. Pharmacokinetics and pharmacodynamics of monoclonal antibodies for asthma treatment. Expert Opin Drug Metab Toxicol. 2019:15:113-120.

8. Goldman M, Hirsch I, Zangrilli JG, Newbold P, Xu X. The association between blood eosinophil count and benralizumab efficacy for patients with severe, uncontrolled asthma: subanalyses of the Phase III SIROCCO and CALIMA studies. Curr Med Res Opin. 2017;33:1605-1613.

9. O'Quinn S, Xu X, Hirsch I. Daily patient-reported health status assessment improvements with benralizumab for patients with severe, uncontrolled eosinophilic asthma. J Asthma Allergy. 2019;12:21-33.

10. Dunican EM, Fahy JV. Asthma and corticosteroids: time for a more precise approach to treatment. Eur Respir J. 2017:49:1701167.

11. Pazdrak K, Moon Y, Straub C, Stafford S, Kurosky A. Eosinophil resistance to glucocorticoid- induced apoptosis is mediated by the transcription factor NFIL3. Apoptosis. 2016;21:421-431.

12. Molfino NA, Gossage D, Kolbeck R, Parker JM, Geba GP. Molecular and clinical rationale for therapeutic targeting of interleukin- 5 and its receptor Clin Exp Allergy. 2012;42:712-737.

13. Varricchi G, Bagnasco D, Borriello F, Heffler E, Canonica GW. Interleukin-5 pathway inhibition in the treatment of eosinophilic respiratory disorders: Evidence and unmet needs. Curr Opin Allergy Clin Immunol. 2016:16:186200

14. Pham TH, Damera G, Newbold P, Ranade K. Reductions in eosinophi biomarkers by benralizumab in patients with asthma. Respir Med 2016;111:21-29

15. O'Quinn S, Xu X, Hirsch I. Daily patient-reported health status assessment improvements with benralizumab for patients with severe, uncontrolled eosinophilic asthma. J Asthma Allergy. 2019;12:21-33.

16. van Dijk BCP, Svedsater H, Heddini A, Nelsen L, Balradj JS, Alleman C. Relationship between the Asthma Control Test (ACT) and other outcomes: a targeted literature review. BMC Pulm Med. 2020;20:79. 